\title{
BMJ Open Are physical factors associated with poor prognosis following a whiplash trauma?: a protocol for a systematic review and data synthesis
}

\author{
Ahmed Alalawi (D) , ${ }^{1,2}$ Alessio Gallina, ${ }^{1}$ Michele Sterling, ${ }^{3}$ Deborah Falla (D) ${ }^{1}$
}

To cite: Alalawi A, Gallina A, Sterling M, et al. Are physical factors associated with poor prognosis following a whiplash trauma?: a protocol for a systematic review and data synthesis. BMJ Open 2019;9:e033298. doi:10.1136/ bmjopen-2019-033298

- Prepublication history for this paper is available online. To view these files, please visit the journal online (http://dx.doi org/10.1136/bmjopen-2019033298).

Received 29 July 2019

Revised 03 October 2019

Accepted 16 October 2019

A Check for updates

(c) Author(s) (or their employer(s)) 2019. Re-use permitted under CC BY-NC. No commercial re-use. See rights and permissions. Published by BMJ.

${ }^{1}$ Centre of Precision Rehabilitation for Spinal Pain (CPR Spine), School of Sports, Exercise and Rehabilitation Sciences, University of Birmingham, Birmingham, UK ${ }^{2}$ Physical Therapy Department, College of Applied Medical

Sciences, Umm Al-Qura

University, Makkah, Saudi Arabia

${ }^{3}$ RECOVER Injury Research

Centre, NHMRC Centre of

Research Excellence in Road

Traffic Injury Recovery, The

University of Queensland,

Brisbane, Queensland, Australia

Correspondence to

Mr Ahmed Alalawi;

amalawi@uqu.edu.sa

\section{ABSTRACT}

Introduction Mitigating the transition from acute to chronic whiplash-associated disorders (WAD) is fundamental, and this could be achieved through early identification of individuals at risk. Several physical factors such as angular velocity, smoothness of neck movement and coactivation of neck flexors and extensors, have been observed in patients with WAD, but their predictive ability after a whiplash injury have not been considered in previous reviews. Therefore, the aim of the current protocol is to outline the protocol for a systematic review that synthesises the current evidence of which physical factors can predict ongoing pain and disability following a whiplash trauma.

Methods and analysis Two independent reviewers will search for studies in several electronic databases including MEDLINE, Embase, CINAHL, PsycINF0, Scopus and Web of Science as well as grey literature. Observational cohort studies will be considered if they involve participants with acute WAD followed for at least 3 months post-injury. Studies will be required to assess the prognostic ability of one or more physical factors that directly involve a body function and/or structure and can be measured objectively. Further, patient-reported outcomes of physical function will be considered. The primary outcome for this review is Neck Disability Index, while all other validated measures will be considered as secondary outcomes. Risk of bias across individual studies will be assessed using the Quality In Prognostic Studies tool along with the Grades of Recommendation, Assessment, Development and Evaluation method to assess the quality of evidence. A meta-analysis will be conducted depending on homogeneity and the number of available studies. If appropriate, data will be pooled and presented as odds ratios, otherwise, a qualitative synthesis will be conducted. Ethics and dissemination Ethical approval is not required for this systematic review. The result from this review will be published in peer-reviewed journals. PROSPERO registration number CRD42019122559

\section{INTRODUCTION}

Whiplash is a term used to describe an injury mechanism associated with a sudden forward and backward movement of the head that is usually due to car collision. ${ }^{1}$ This sudden impact may result in injuries to multiple

\section{Strengths and limitations of this study}

- This systematic review will be the first to rigorously summarise and evaluate the current body of evidence regarding the predictive ability of physical factors following a whiplash trauma, using a combination of clinical and patient-reported outcome measures.

- Several methodological limitations across included studies are anticipated such as substantial heterogeneity, high risk of bias, variabilities in whiplash severity and source of participants, which may lead to potential difficulty in interpreting and applying the results.

- This review will consider studies reported in English language only and therefore may miss studies published in other languages.

structures in the neck, ${ }^{1}$ which lead to the development of a wide range of clinical manifestations commonly termed as whiplashassociated disorder (WAD). ${ }^{1}$ WAD has a substantial socioeconomic burden, ${ }^{2}$ with costs to the UK economy $\sim £ 3$ billion per year. ${ }^{3}$ It is a source of disability ${ }^{45}$ with common negative consequences including limited work ability, fatigue, restricted participation in sports, depression, frustration and anger. ${ }^{67}$

The rate of transition from acute to chronic WAD is high. It has been found that $50 \%$ of patients with acute WAD develop chronic WAD,${ }^{589}$ a condition that tends to be resistant to treatment with limited evidence of effective interventions. ${ }^{1011}$ Additionally, there is a large variability between individuals in how they respond to a specific intervention. ${ }^{12}$ For example, only up to $44 \%$ of patients with chronic WAD reported a significant reduction in pain following a 12-week programme of specific neck exercise. ${ }^{12}$ Due to this general lack of responsiveness to interventions, mitigating the transition to chronic WAD, in the first place, is fundamental. This could be achieved through early identification 
of physical factors that increase the risk of developing persistent symptoms, among whom, a better allocation of treatments could be prescribed. ${ }^{13}$

Many syntheses have been conducted in the field of WAD prognosis aiming to identify factors that are associated with the outcomes following a whiplash trauma. To this end, 12 systematic reviews focused on prognosis following a whiplash injury were found, ${ }^{5814-23}$ and covered a myriad factors including social, psychological and physical factors. A review of these reviews found that an initial high level of neck pain and disability following a whiplash injury is associated with poor outcome. ${ }^{2425}$ However, there is still inconsistency in the reported evidence concerning the predictive ability of other factors including post-injury anxiety, catastrophizing, cold hyperalgesia, legal and compensation factors, WAD grading and early healthcare use. ${ }^{24}$ Additionally, there is inconsistency in the results among systematic reviews regarding the predictive ability of physical factors (eg, restricted cervical range of motion $(\mathrm{ROM})$ ).

Qualitative synthesis from systematic reviews showed limited evidence about the association between restricted cervical ROM and persistent disability, ${ }^{16} 2122$ whereas no such association was found in another review. ${ }^{8}$ This was also shown in a meta-analysis of six cohorts investigating the prognostic ability of restricted ROM on persistent neck pain and disability. ${ }^{19}$ Due to this controversy and to the fact that it has been 6 years since the last systematic review on physical factors,${ }^{16}$ a systematic review is needed.

Several other physical factors have been observed to be impaired in patients with WAD, yet they have not considered in current reviews. These include changes in motor function and muscle behaviour such as decreased maximum angular velocity, ${ }^{26}{ }^{27}$ larger jerk index (a measure of the smoothness of neck movement) ${ }^{27}$ and increased coactivation of neck flexors and extensors. ${ }^{28}$ The presence of these adaptions was also observed in experimental pain studies, ${ }^{29-36}$ where patients injected with a hypersaline solution inducing an immediate pain similar to a traumatic event. Besides, patient-reported outcome measures to assess physical function could be useful in predicting outcomes following a whiplash trauma.

Physical functioning was recommended by an international multidisciplinary panel as one of the core domains to be reported in clinical studies involving patients with WAD, ${ }^{37}$ and low back pain. ${ }^{38-40}$ 'Physical function is a broad domain that can encompass various aspects of a person's life including ability to carry out daily activities, eg, household tasks, recreational activities or self-care to specific strength, endurance and functional capacity'. ${ }^{37}$ Yet, there is no consensus on the measurement instruments of physical functioning in the field on whiplash. Recommendations on selecting measurement interments to measure physical functioning were formulated in individuals with low back pain, ${ }^{40}$ including Oswestry Disability Index V.2.1a and 24-item Roland-Morris Disability Questionnaire.
Therefore, the aim of this systematic review is twofold: (1) To inform and summarise the objective physical measures that have been used to date in prognostic research in this population and (2) To synthesise the evidence regarding the predictive ability of these physical factors on neck pain and disability in individuals following a whiplash trauma.

\section{METHODS AND ANALYSIS}

\section{Registration and methodology}

This is a protocol for conducting a systematic review aiming to identify whether physical factors are associated with ongoing pain and disability following a whiplash trauma. The protocol was planned according to the guidelines proposed by Moons et al for conducting prognostic reviews, ${ }^{41}$ and reported according to the guidelines from Preferred reporting items for systematic review and meta-analysis protocols, ${ }^{42}$ the Cochrane Handbook ${ }^{43}$ and the Cochrane Back Review Group guidelines. ${ }^{44}$

\section{Protocol registration}

The protocol of this review was registered on PROSPERO (International Prospective Register of Systematic Reviews).

\section{ELIGIBILITY CRITERIA \\ Inclusion criteria}

\section{Studies}

Observational studies will be included if they describe the association between physical factors and prognosis in individuals who have sustained a whiplash injury and who have been followed up over time for a minimum of 3 months. Other study designs such as case reports or case-control studies will be excluded from this review, as well as any review articles, letters, editorials, conference proceedings and studies with only abstracts. Only articles published in English will be considered.

\section{Participants}

Studies will be included if they involve populations with the characteristics below:

1. Participants with acute WAD ( $<6$ weeks) attributed to a motor traffic collision or sports injury and classified as grade I, II or III on Quebec Task Force (QTF) classification. ${ }^{1}$ If the cause of acute WAD was not specified, the paper will be considered as well.

2. Participants were followed up over time for at least 3 months. Studies with different time-points beyond 3 months will be considered.

3. Aged $>16$ years old

\section{Exposure or intervention (potential prognostic factors)}

Studies will be required to assess the prognostic ability of one or more physical factors measured at baseline regardless of the measurement used. Because there is no consensus on the definition of physical factors specifically 
in the field of WAD, physical factors will be selected, for the purpose of this review, if they directly involve a body function and/or structure and can be measured objectively. These include neck self-reported measures of physical functioning (eg, patient specific functional scale, physical component of the SF-36), joint position sense, movement sense, proprioception, onset and amplitude of muscle activation, range of neck movement, quality of neck movement, velocity of neck movement, tests of eye movement control, neck muscle strength and endurance, neck muscle fatigue, balance and the morphology of the cervical spine muscles. Any spinal structural changes or findings in X-ray will not be considered in this review.

\section{Outcome}

The primary outcome of interest is the Neck disability Index ${ }^{45}$ measured at least at 3 months follow-up. All other validated outcomes that were used in primary studies to describe the association between physical factors and an outcome will be included in the review and considered as secondary outcomes of interest such as pain intensity, psychological status, health-related quality of life, selfrated recovery and functional recovery.

\section{Exclusion criteria}

Other study designs such as case reports or case-control studies will be excluded from this review, as well as any review articles, letters, editorials, conference proceedings and studies with only abstracts. Only articles published in English will be considered. Also, studies will be excluded if they include patients with previous cervical pain, surgery or combine subjects with WAD and other musculoskeletal injuries.

\section{Search strategy}

Several databases will be searched from 1995 to August 2019 including MEDLINE (OVID), Embase (OVID), Cumulative Index to Nursing and Allied Health Literature (CINAHL), PsycINFO (OVID), Scopus and Web of Science as well as grey literature through Zetoc database which includes any document that usually not published commercially as a peer-reviewed article. ${ }^{46}$ The identified key words in box 1 will be used to search for relevant studies including unpublished articles. We limit our search to 1995 as the standardised definition of WAD was provided by the QTF monograph, an approach used previously in a systematic reviews. ${ }^{14}{ }^{19}$ Notable authors in the field will be contacted to identify relevant unpublished literature which is currently in preparation. Moreover, reference lists of retrieved individual studies will be screened for relevant studies as well as any relevant published reviews on prognosis in WAD to ensure all related studies have been identified. The searching process will be limited to the English language.

A combination of free text and Medical Subject Heading (MeSH) will be used to retrieve all related studies. The related search terms related to WAD, whiplash trauma and physical prognostic factors have been informed from
Box 1 Example of searching strategy for MEDLINE (OVID) electronic database

\section{Search terms}

1. Whiplash Injuries/pa, pp, rh, th [Pathology, Physiopathology, Rehabilitation, Therapy]

2. Whiplash Injur*.mp.

3. (Whiplash or WAD).mp.

4. $\left(\right.$ Motor adj accident $\left.{ }^{\star}\right) \cdot \mathrm{mp}$.

5. (Motor adj crash*).mp.

6. Neck Injuries/pa, pp, rh, th [Pathology, Physiopathology, Rehabilitation, Therapy]

7. (Neck adj2 injurie*).mp.

8. (Neck adj2 Sprain*).mp.

9. (Neck adj2 strain*).mp.

10. $\mathrm{OR} / 1-9$

11. "cervical dysfunction*".mp.

12. (Neck adj2 dysfunction*).mp

13. ('Joint adj2 sense').mp.

14. joint position error'.mp.

15. sensorimotor control.mp

16. motor control.mp.

17. motor system dysfunction.mp.

18. sensorimotor dysfunction $\$ . m p$.

19. (neck adj3 sense).mp.

20. Proprioception/ or neck sense.mp.

21. neuromuscular control.mp.

22. Muscle* activation*.mp.

23. Co?activation*.mp.

24. movement* quality.mp.

25. (quality adj movement).mp.

26. (Neck adj2 motion).mp.

27. angular velocity.mp. or Rotation/

28. Eye Movements/ or Pursuit, Smooth/ or 'movement smooth*'.mp. or Motion Perception/

29. ('neck adj3 strength').mp.

30. ('neck adj3 endurance').mp.

31. ('Deep adj3 muscle $\left.{ }^{\star \prime}\right) \cdot m p$.

32. ('superficial adj3 muscle $\left.{ }^{\star \prime}\right) \cdot m p$.

33. onset of activation $\$$ '.mp.

34. Somatosensory Disorders/et, pp, rh [Etiology, Physiopathology, Rehabilitation]

35. ('Smooth adj3 movement').mp.

36. ('Alter* adj3 strategy $\left.{ }^{\star \prime}\right) \cdot m p$.

37. Isometric Contraction/ or Co?contraction*.mp.

38. Fatigue/ or Muscle Fatigue/ or Muscle* fatigue.mp.

39. Muscle, Skeletal/ or Adaptation, Physiological/ or Peripheral adaptation.mp.

40. Fatty infiltration*.mp. or Magnetic Resonance Imaging/

41. physical measure*

42. Self adj3 measure*

43. Patient ${ }^{\star}$ adj3 measure*

44. Physical adj function*

45. Patient* adj2 outcome*

46. Muscular Atrophy/ or Immobilization/ or Muscle* disuse.mp.

47. Balance.mp. or POSTURAL BALANCE/

48. Atrophy.mp. or ATROPHY/

49. $\mathrm{OR} / 11-48$

50. Validat\$.mp. or Predict\$.ti. or Rule\$.mp. or (Predict\$ and (Outcome\$ or Risk\$ or Model\$)).mp. or ((History or Variable\$ or Criteria or Scor\$ or Characteristic\$ or Finding\$ or Factor\$) and

Continued 


\section{Box 1 Continued}

(Predict\$ or Model\$ or Decision\$ or Identif\$ or Prognos\$)).mp. or (Decision\$.mp. and ((Model\$ or Clinical\$).mp. or Logistic Models/)) or (Prognostic and (History or Variable\$ or Criteria or Scor\$ or Characteristic $\$$ or Finding $\$$ or Factor $\$$ or Model\$)).mp.

51. (Predict* or Predictive value of tests or Scor* or Observ* or Observer variation).mp.

52. ('Stratification' or 'ROC Curve' or 'Discrimination' or 'Discriminate' or 'c-statistic' or 'c statistic' or 'Area under the curve' or 'AUC' or 'Calibration' or 'Indices' or 'Algorithm' or 'Multivariable').mp.

53. Risk Factors/ or Predict\$ factor\$.mp.

54. Predict\$ variable\$.mp.

55. Prognos\$ factor\$.mp.

56. Prognos $\$$ variable\$.mp.

57. (Candidate adj3 factor ${ }^{\star}$ ).mp.

58. Candidate predictor ${ }^{*} . \mathrm{mp}$.

59. Prognosis/or Progn ${ }^{\star} . \mathrm{mp}$.

60. Predic*.mp

61. $\mathrm{OR} / 50-60$

62. 10 AND 49 AND 61

previously published reviews in prognosis following WAD trauma, ${ }^{814-16181924}$ and from our scoping searches. To increase the sensitivity of retrieving all related prognosis studies, the proposed prognosis filters that identified by Geersing $e t$ al. $^{47}$ will be utilised in addition to other filters identified previously. ${ }^{48}{ }^{49}$ The use of relevant phrases and $\mathrm{MeSH}$ terms are expected to be varied between databases. A total number of hits in each database and the excluded papers with the reasons will be reported in the main review. The search will be conducted by the lead author (AA) and has been informed by subject specific expertise and the completion of scoping searches. An example search in MEDLINE (OVID) is demonstrated in box 1.

\section{Data management}

Relevant citations and abstracts will be managed using EndNote V.X9 (Clarivate Analytics) software programme during the process of storing, removing duplicates and screening processes. Relevant forms will be developed to aid the screening process. A Microsoft Excel spreadsheet will be used to store all the extracted data.

\section{Study selection}

Once duplicates have been removed, two reviewers will independently conduct searches and screen titles and abstracts of the studies against the predetermined eligibility criteria to avoid missing related studies. Full text of the studies will be retrieved if eligibility were met or in case a conclusion could not be possible to be made based on the title or abstract. In the case where multiple papers were published from the same cohort that investigated the same predictor, the original cohort will be selected and then extracted for this review, an approach was used previously in published systematic reviews. ${ }^{14} 1922$ The same two reviewers will screen full texts eligibility and review relevant references lists. A third reviewer (DF) will be consulted to resolve any agreement by discussion if consensus could not be reached.

\section{Data collection process}

Data will be extracted using a modified data extraction form. The form will be reviewed and finalised through a pilot test of a small number of eligible studies during the process of data extraction. Both reviewers will extract the data independently, who will then meet to check the accuracy of the extracted data. A third reviewer (DF) will mediate any disagreement in data extraction.

\section{Data items}

The data extraction items were informed by the CHecklist for critical Appraisal and data extraction for systematic Reviews of prediction Modelling Studies. ${ }^{41}$ Although this tool was designed for reviews of primary prediction modelling studies, some domains have been selected to inform selecting data items of this review. The following data will be extracted from each study: authors and year of publication, study location, study design, participants characteristics, outcomes of interest, candidate predictors, sample size, length of follow-up, items associated with risk of bias, summary statistics and methods for statistical analysis. The corresponding author of the original studies will be contacted for clarification and missing data if required. If no response is received from a corresponding author and the inquiry affects the eligibility of the study, it will be excluded from this review.

\section{Risk of bias}

To evaluate the risk of bias of included individual studies, the Quality In Prognostic Studies tool ${ }^{50}$ will be used. The tool was designed to assess bias in review questions related to prognostic factors ${ }^{51}$ and showed acceptable inter-rater reliability. ${ }^{50}$ It considers six domains when assessing bias in prognostic studies; study participation, study attrition, prognostic factor measurement, confounding measurement and account, outcome measurement and analysis and reporting. ${ }^{51}$ Each risk of bias domain is rated as a 'high', 'moderate' or 'low' based on consensus judgement from at least two assessors. ${ }^{50}$ To assess the overall risk of bias of individual study, a study gets overall of low risk of bias, when all six domains rated as a low risk, while study judged as having a high risk a bias if $\geq 1$ domain assessed as a high risk of bias. Two reviewers will assess the risk of bias independently. Any disagreement will be resolved by discussion or by a third reviewer (DF) if consensus could not be reached.

\section{Quality of evidence}

The overall quality of evidence for a prognostic factor per outcome across studies will be assessed using The Grading of Recommendations, Assessment, Development and Evaluation (GRADE) approach. ${ }^{52}$ The GRADE approach criteria was modified to be used in prognostic factor research. ${ }^{5354}$ The adapted GRADE for prognostic factors research includes six factors that decrease the quality of evidence including 'phase of investigation', 'study limitations', 'inconsistency', 'indirectness, imprecision', 'publication bias', while two 
factors increase the quality 'moderate or large effect size', 'exposure-response gradient'. ${ }^{53}$ The phase of investigation GRADE domain is a district for prognostic studies phase 3 and phase 2 considered the highest quality of evidence. ${ }^{53}$ The GRADE system will be applied to assess the overall quality of evidence of confirmed prognostic factors generated from univariate results, as used previously. ${ }^{55}$

\section{Data synthesis and analysis}

A quantitative synthesis will be planned depending on homogeneity between included studies. If meta-analysis is not possible, a qualitative synthesis of the results will be conducted.

\section{Summary statistics}

When outcomes are binary, they expected to be presented as OR in primary studies calculated by the logistic regression model. ${ }^{56}$ Therefore, pooled ORs will be used to calculate the effect estimate of a prognostic ability of predictors on an outcome of interest. Since it is expected that primary studies have used different effect estimators to calculate the prognostic ability of a factor on an outcome, some statistical conversions may be required. For example, if OR and risk ratio (RR) were not provided, they could be estimated manually based on the number of events among two comparative groups. ${ }^{57}$ In case where potential predictors or outcome are continuous variables, the mean difference or adjusted mean difference will be used to represent a summary effect. ${ }^{57}$ If needed, the mean difference may be converted into standardised mean difference when combined. ${ }^{58}$ If estimation from available data is unfeasible, authors will be contacted to provide data. If no response is received, the study will be excluded from the meta-analysis. All statistical conversions will be reported in the main manuscript.

\section{Data synthesis}

The results will be pooled if an association between an outcome and specific prognostic factor was presented by the same summary statistics in two or more cases. OR or $\mathrm{RR}$ will be summarised separately if the outcome is binary whereas continuous outcome will be combined using mean difference or standardised mean difference. When continuous variables are presented using the median instead of the mean, they will not be combined and handled as it is. ${ }^{57}$

Because this review includes studies with univariate and multivariate analysis, it is expected that some studies will report univariate analysis and others with multivariate analysis. In this case, only the unadjusted estimates of prognostic factors will be pooled. This is because of the confounding effect of factors within a multivariate model which could give misleading results; therefore, effect estimates from multivariate models will be summarised qualitatively.

\section{Meta-analysis}

If meta-analysis is feasible, the random-effect model (DerSimonian and Laird method) will be conducted ${ }^{58}$ utilising the Statistical Software Package, Review Manager V.5.3. ${ }^{59}$
A significant univariate association between a factor and outcome will be considered present if the reported $\mathrm{p}$ value is $<0.05$ or $95 \%$ of CIs of OR or similar statistical methods do not get below one. ${ }^{8}$ If combined results are presented, the $95 \%$ prediction interval will be calculated. ${ }^{60}$

\section{Heterogeneity}

Heterogeneity of the pooled estimate will be assessed using $Q$ statistic and the $\mathrm{I}^{2}$ test. Statistical heterogeneity will be considered significant between studies if $p<0.1$, as this test has low power. ${ }^{61}$ Beside the $\mathrm{Q}$ statistic and to measure the magnitude of heterogeneity, the $\mathrm{I}^{2}$ test will be used which gives a score range from $0 \%$ to $100 \%$, where scores from $(0 \%$ to $30 \%)$, ( $30 \%$ to $50 \%)$, (50\% to $70 \%$ ) and (70\% to $100 \%)$ indicates low, moderate, considerable and substantial heterogeneity, respectively. ${ }^{58}$ In the case of low heterogeneity, the fixed-effect model will be used as it gives weight better than the randomeffect model, otherwise, the random effect model will be used. ${ }^{62}$ However, both tests may be affected by the number of included studies which could not detect heterogeneity in some cases. Because of this, heterogeneity will also be investigated using forest plots to see if the estimated effect overlaps with all CIs across studies. If heterogeneity is present, further exploration will be performed including subgroup analysis and sensitivity analysis.

\section{Subgroup analysis}

Subgroup analysis is planned to clarify the source of heterogeneity if present between studies. High heterogeneity among studies is more likely to be present. A priori potential sources of heterogeneity could arise from WAD grade, study design, source of participants, follow-up time.

\section{Sensitivity analysis}

Sensitivity analysis will be performed to examine the robustness of the results by including studies with only high-quality.

\section{Reporting bias}

A funnel plot will be used to examine publication bias within studies. Also, Egger's test will be used statistically to examine publication bias, ${ }^{63}$ with statistical power set at $\mathrm{p}<0.1$ due to the low power of this test, which shows evidence of publication bias. If it is suspected, the trim and fill method will be applied. ${ }^{64}$

\section{Confirmation of prognostic factors}

The overall decision of judging whether a factor is prognostic will be based on two criteria, an approach used previously. ${ }^{55}$ First, the same factor must show statistical univariate association with an outcome in at least $75 \%$ of all included studies. Second, the effect of prognostic factors is consistently in the same direction of effect across all studies. Further, if a multivariate analysis about the prognostic ability of a factor is available, it will be used to confirm such association. These criteria would allow a quantitative and robust methodology which allows replicable results. 


\section{Patients and public involvement}

The research question in this study was developed following consultations with patients. Patients will not be involved in the analysis and data collection of the systematic review.

\section{Ethics and dissemination}

No ethical approval is required for this systematic review, as there is no patient data being collected. The result of this review will be published in peer-reviewed journals and presented in national and international conferences.

\section{Implication of results}

The results obtained from this review will have implications for understanding the recovery after whiplash trauma. In particular, information on physical factors following whiplash injury will be synthesised and their predictive ability will be demonstrated, if present. This will inform future research agenda on the predictive ability of physical factors in patients with acute whiplash. Particularly, future studies could be designed to create and test screening tools to categorise patients with acute WAD into low risk and high risk of developing persistent symptoms, which will inform early intervention and management. Additionally, intervention resources could be targeted towards those with the risk of poor outcomes which could mitigate their risk of developing ongoing symptoms, informing health policy and clinical management. Although this systematic review focuses on physical measures only, the findings will be discussed with consideration of the current knowledge on which psychosocial factors can predict ongoing pain and disability following whiplash trauma.

\section{Twitter Ahmed Alalawi @alawi_pt and Deborah Falla @Deb_Falla}

Contributors All authors contributed to the focus of the systematic review topic. AA is a PhD student with DF as Lead Supervisor and AG as Co-Supervisor. AA drafted the initial protocol with guidance from DF at all stages. MS and AG provided feedback on manuscript drafts and all authors approved the final version for publication. DF is guarantor.

Funding The authors have not declared a specific grant for this research from any funding agency in the public, commercial or not-for-profit sectors.

Competing interests None declared.

Patient consent for publication Not required.

Provenance and peer review Not commissioned; externally peer reviewed.

Open access This is an open access article distributed in accordance with the Creative Commons Attribution Non Commercial (CC BY-NC 4.0) license, which permits others to distribute, remix, adapt, build upon this work non-commercially, and license their derivative works on different terms, provided the original work is properly cited, appropriate credit is given, any changes made indicated, and the use is non-commercial. See: http://creativecommons.org/licenses/by-nc/4.0/.

\section{ORCID iDs}

Ahmed Alalawi http://orcid.org/0000-0001-5667-8150

Deborah Falla http://orcid.org/0000-0003-1689-6190

\section{REFERENCES}

1 Spitzer WO, Skovron ML, Salmi LR, et al. Scientific monograph of the Quebec Task Force on Whiplash-Associated Disorders: redefining "whiplash" and its management. Spine 1995;20:1S-73.

2 Holm LW, Carroll LJ, Cassidy JD, et al. The burden and determinants of neck pain in whiplash-associated disorders after traffic collisions: results of the bone and joint decade 2000-2010 Task force on neck pain and its associated disorders. J Manipulative Physiol Ther 2009;32:S61-9.

3 Melody J. Whiplash associated disorder training pack. London: British Association for Accident and Emergency Medicine, 2003.

4 Carroll LJ, Ferrari R, Cassidy JD, et al. Coping and recovery in whiplash-associated disorders: early use of passive coping strategies is associated with slower recovery of neck pain and painrelated disability. Clin J Pain 2014;30:1-8.

5 Carroll LJ, Holm LW, Hogg-Johnson S, et al. Course and prognostic factors for neck pain in whiplash-associated disorders (WAD): results of the bone and joint decade 2000-2010 Task force on neck pain and its associated disorders. J Manipulative Physiol Ther 2009;32:S97-107.

6 Hoving JL, O'Leary EF, Niere KR, et al. Validity of the neck disability index, Northwick Park neck pain questionnaire, and problem elicitation technique for measuring disability associated with whiplash-associated disorders. Pain 2003;102:273-81.

7 Pinfold M, Niere KR, O'Leary EF, et al. Validity and internal consistency of a whiplash-specific disability measure. Spine 2004;29:263-8.

8 Kamper SJ, Rebbeck TJ, Maher CG, et al. Course and prognostic factors of whiplash: a systematic review and meta-analysis. Pain 2008;138:617-29.

9 Hendriks EJM, Scholten-Peeters GGM, van der Windt DAWM, et al. Prognostic factors for poor recovery in acute whiplash patients. Pain 2005;114:408-16.

10 Teasell RW, McClure JA, Walton D, et al. A research synthesis of therapeutic interventions for whiplash-associated disorder (WAD): part 4 - noninvasive interventions for chronic WAD. Pain Res Manag 2010;15:313-22.

11 Teasell RW, McClure JA, Walton D, et al. A research synthesis of therapeutic interventions for whiplash-associated disorder (WAD): part 5 - surgical and injection-based interventions for chronic WAD. Pain Res Manag 2010;15:323-34.

12 Ludvigsson ML, Peterson G, O'Leary S, et al. The effect of neckspecific exercise with, or without a behavioral approach, on pain, disability, and self-efficacy in chronic whiplash-associated disorders: a randomized clinical trial. Clin J Pain 2015;31:294-303.

13 Jull GA, Söderlund A, Stemper BD, et al. Toward optimal early management after whiplash injury to lessen the rate of transition to chronicity: discussion paper 5. Spine 2011;36:S335-42.

14 Walton DM, Macdermid JC, Giorgianni AA, et al. Risk factors for persistent problems following acute whiplash injury: update of a systematic review and meta-analysis. J Orthop Sports Phys Ther 2013;43:31-43.

15 Li Q, Shen H, Li M. Magnetic resonance imaging signal changes of alar and transverse ligaments not correlated with whiplashassociated disorders: a meta-analysis of case-control studies. Eur Spine J 2013;22:14-20.

16 Daenen L, Nijs J, Raadsen B, et al. Cervical motor dysfunction and its predictive value for long-term recovery in patients with acute whiplash-associated disorders: a systematic review. J Rehabil Med 2013;45:113-22.

17 Spearing NM, Connelly LB, Gargett S, et al. Does injury compensation lead to worse health after whiplash? A systematic review. Pain 2012;153:1274-82.

18 Goldsmith R, Wright C, Bell SF, et al. Cold hyperalgesia as a prognostic factor in whiplash associated disorders: a systematic review. Man Ther 2012;17:402-10.

19 Walton DM, Pretty J, MacDermid JC, et al. Risk factors for persistent problems following whiplash injury: results of a systematic review and meta-analysis. J Orthop Sports Phys Ther 2009;39:334-50.

20 Williamson E, Williams M, Gates S, et al. A systematic literature review of psychological factors and the development of late whiplash syndrome. Pain 2008;135:20-30.

21 Williams M, Williamson E, Gates S, et al. A systematic literature review of physical prognostic factors for the development of late whiplash syndrome. Spine 2007;32:E764-80.

22 Scholten-Peeters GGM, Verhagen AP, Bekkering GE, et al. Prognostic factors of whiplash-associated disorders: a systematic review of prospective cohort studies. Pain 2003;104:303-22.

23 Côté P, Cassidy JD, Carroll L, et al. A systematic review of the prognosis of acute whiplash and a new conceptual framework to synthesize the literature. Spine 2001;26:E445-58.

24 Sarrami P, Armstrong E, Naylor JM, et al. Factors predicting outcome in whiplash injury: a systematic meta-review of prognostic factors. $J$ Orthopaed Traumatol 2017;18:9-16.

25 Walton DM, Carroll LJ, Kasch H, et al. An overview of systematic reviews on prognostic factors in neck pain: results from the International collaboration on neck pain (icon) project. Open Orthop J 2013;7:494-505. 
26 Baydal-Bertomeu JM, Page AF, Belda-Lois JM, et al. Neck motion patterns in whiplash-associated disorders: quantifying variability and spontaneity of movement. Clin Biomech 2011;26:29-34.

27 Sjölander P, Michaelson P, Jaric S, et al. Sensorimotor disturbances in chronic neck pain--range of motion, peak velocity, smoothness of movement, and repositioning acuity. Man Ther 2008;13:122-31.

28 Fernández-de-las-Peñas C, Falla D, Arendt-Nielsen L, et al. Cervical muscle co-activation in isometric contractions is enhanced in chronic tension-type headache patients. Cephalalgia 2008;28:744-51.

29 Falla D, Farina D, Dahl MK, et al. Muscle pain induces taskdependent changes in cervical agonist/antagonist activity. J Appl Physiol 2007;102:601-9.

30 Madeleine P, Leclerc F, Arendt-Nielsen L, et al. Experimental muscle pain changes the spatial distribution of upper trapezius muscle activity during sustained contraction. Clin Neurophysiol 2006;117:2436-45.

31 Madeleine P, Mathiassen SE, Arendt-Nielsen L. Changes in the degree of motor variability associated with experimental and chronic neck-shoulder pain during a standardised repetitive arm movement. Exp Brain Res 2008;185:689-98.

32 Muceli S, Falla D, Farina D. Reorganization of muscle synergies during multidirectional reaching in the horizontal plane with experimental muscle pain. J Neurophysiol 2014;111:1615-30.

33 Smith M, Coppieters MW, Hodges PW. Effect of experimentally induced low back pain on postural sway with breathing. Exp Brain Res 2005;166:109-17.

34 Tucker KJ, Hodges PW. Changes in motor unit recruitment strategy during pain alters force direction. Eur J Pain 2010;14:932-8.

35 Hug F, Hodges PW, Tucker K. Task dependency of motor adaptations to an acute noxious stimulation. J Neurophysiol 2014;111:2298-306.

36 Gizzi L, Muceli S, Petzke F, et al. Experimental muscle pain impairs the synergistic modular control of neck muscles. PLoS One 2015;10:e0137844.

37 Chen K, Andersen T, Carroll L, et al. Recommendations for core outcome domain set for Whiplash-Associated disorders (CATWAD). Clin J Pain 2019;35:727-36.

38 Chiarotto A, Deyo RA, Terwee CB, et al. Core outcome domains for clinical trials in non-specific low back pain. Eur Spine $J$ 2015;24:1127-42.

39 Turk DC, Dworkin RH, Allen RR, et al. Core outcome domains for chronic pain clinical trials: IMMPACT recommendations. Pain 2003;106:337-45.

40 Chiarotto A, Boers M, Deyo RA, et al. Core outcome measurement instruments for clinical trials in nonspecific low back pain. Pain 2018;159:481.

41 Moons KGM, de Groot JAH, Bouwmeester W, et al. Critical appraisal and data extraction for systematic reviews of prediction modelling studies: the charms checklist. PLoS Med 2014;11:e1001744.

42 Moher D, Shamseer L, Clarke M, et al. Preferred reporting items for systematic review and meta-analysis protocols (PRISMA-P) 2015 statement. Syst Rev 2015;4:1.

43 Higgins JP, Green S. Cochrane Handbook for systematic reviews of interventions 2008
44 Furlan AD, Pennick V, Bombardier C, et al. 2009 updated method guidelines for systematic reviews in the Cochrane back review group. Spine 2009;34:1929-41.

45 Vernon $\mathrm{H}$, Mior $\mathrm{S}$. The neck disability index: a study of reliability and validity. J Manipulative Physiol Ther 1991;14:409-15.

46 Braithwaite J, Zurynski Y, Ludlow K, et al. Towards sustainable healthcare system performance in the 21st century in high-income countries: a protocol for a systematic review of the grey literature. BMJ Open 2019;9:e025892.

47 Geersing G-J, Bouwmeester W, Zuithoff P, et al. Search filters for finding prognostic and diagnostic prediction studies in MEDLINE to enhance systematic reviews. PLoS One 2012;7:e32844.

48 Wilczynski NL, Haynes RB, Hedges Team. Developing optimal search strategies for detecting clinically sound prognostic studies in MEDLINE: an analytic survey. BMC Med 2004:2:23.

49 Wilczynski NL, Haynes RB. Optimal search strategies for detecting clinically sound prognostic studies in EMBASE: an analytic survey. $J$ Am Med Inform Assoc 2005;12:481-5.

50 Hayden JA, van der Windt DA, Cartwright JL, et al. Assessing bias in studies of prognostic factors. Ann Intern Med 2013;158:280-6.

51 Hayden JA, Côté P, Bombardier C. Evaluation of the quality of prognosis studies in systematic reviews. Ann Intern Med 2006;144:427-37.

52 Group GW. Grading of recommendations assessment, development and evaluation (grade). access date 2012;23.

53 Huguet A, Hayden JA, Stinson J, et al. Judging the quality of evidence in reviews of prognostic factor research: adapting the grade framework. Syst Rev 2013;2:71

54 lorio A, Spencer FA, Falavigna M, et al. Use of grade for assessment of evidence about prognosis: rating confidence in estimates of event rates in broad categories of patients. BMJ 2015;350:h870.

55 Kamiya $\mathrm{H}$, Panlaqui OM. Prognostic factors for acute exacerbation of idiopathic pulmonary fibrosis: protocol for a systematic review and meta-analysis. BMJ Open 2019;9:e028226.

56 Bock JR, Afifi AA. Estimation of probabilities using the logistic model in retrospective studies. Comput Biomed Res 1988;21:449-70.

57 Kamiya H, Panlaqui OM. Prognostic significance of autoantibodies for idiopathic pulmonary fibrosis: protocol for a systematic review. BMJ Open 2018;8:e020862.

58 Higgins J, Green S. Cochrane collaboration: cochrane Handbook for systematic reviews of interventions. Cochrane book series, 2008.

59 Collaboration C. Review manager (RevMan) version 5.3. Copenhagen: The Nordic Cochrane Centre, 2014.

60 Riley RD, Higgins JPT, Deeks JJ. Interpretation of random effects meta-analyses. BMJ 2011;342:d549.

61 Lau J, loannidis JP, Schmid CH. Quantitative synthesis in systematic reviews. Ann Intern Med 1997;127:820-6.

62 Hedges LV, Vevea JL. Fixed- and random-effects models in metaanalysis. Psychol Methods 1998;3:486-504.

63 Egger M, Smith GD, Schneider M, et al. Bias in meta-analysis detected by a simple, graphical test. BMJ 1997;315:629-34.

64 Duval S, Tweedie R. Trim and fill: a simple funnel-plot-based method of testing and adjusting for publication bias in meta-analysis. Biometrics 2000;56:455-63. 